\title{
Median Nucleic Acid Conversion Time and The Clinical and Laboratory Parameters Affecting It in Patients with COVID-19
}

\author{
Kadir Canoğlu ${ }^{1}$, Tayfun Calişkan ${ }^{1}$, and Ecem Sinmez ${ }^{2}$ \\ ${ }^{1}$ Sultan Abdulhamid Han Egitim ve Arastirma Hastanesi \\ ${ }^{2}$ Sultan Abdülhamid Han Training and Research Hospital
}

October 3, 2021

\begin{abstract}
Background: The time for PCR positivity to negativity is defined as nucleic acid conversion time (NCT) and is very important in terminating the isolation of patients and determining infectiousness in patients with COVID-19. Objective: The aim of this study is to determine the median NCT and to evaluate the clinical and laboratory parameters affecting it in patients with COVID-19. Methods: This study included 318 mild to moderate patients with COVID-19 diagnosed with PCR positivity retrospectively. Results: The median NCT was 11 days. Patients were divided into 2 groups as early ( $<11$ days) and late conversion ( $>=11$ days). Older age, sore throat, onset fever, fever 72 hours after hospitalization, history of exposure to SARS-CoV-2 virus without a mask and moderated disease were significantly more common in the late conversion group. In addition, favipiravir use was higher in early conversion group and hydroxychloroquine use was higher in late conversion group. In multivariate analysis, sore throat $(\mathrm{OR}=2.570 ; 95 \% \mathrm{CI}: 1.051-6.284)$ and hydroxychloroquine use $(\mathrm{OR}=3.518,95 \% \mathrm{CI}$ : 1.163-10.635) were independent risk factors for late conversion. Favipiravir use (OR $=0.062,95 \%$ CI: 0.021-0.184) negatively affected the late conversion. Conclusion: NCT was longer in patients with COVID-19 who had sore throat at admission and were treated with hydroxychloroquine instead of favipiravir. Keywords: COVID-19, Nucleic Acids, Polymerase Chain Reaction, SARS-CoV-2.
\end{abstract}

\section{Hosted file}

manuscript.docx available at https://authorea.com/users/405024/articles/540161-mediannucleic-acid-conversion-time-and-the-clinical-and-laboratory-parameters-affecting-it-inpatients-with-covid-19 